\title{
Reinforcement locus and free-operant extinction ${ }^{1}$
}

JOHN J. PORTER

UNIVERSITY OF WISCONSIN-MILWAUKEE

Six groups of rats received either 18 or 96 reinforcements delivered either at the bar, 2 in. from the bar, or 4 in. from the bar. Ss rewarded at the bar extinguished significantly faster after 96 than 18 reinforcements. The converse was true for the other two bar locations. Results were discussed in terms of frustration theory.

Several recent runway studies (cf., Ison, 1962; Ison \& Cook, 1964; North \& Stimmel, 1960) have shown, with other variables held constant, that resistance to extinction is inversely related to the number of reinforcements. This inverse relationship between number of acquisition trials and resistance to extinction has been explained in terms of frustration, which has been assumed to produce competing responses (CRs) which interfere with the previously learned instrumental response (Birch, 1961). The magnitude of these CRs during extinction has been assumed to increase with more reinforcements during acquisition.

In contrast to runway studies, free-operant extinction has appeared to be an increasing function of number of reinforcements (cf., Williams, 1938; Perin, 1942). However, no systematic attempt has been made to resolve the apparent contradiction between freeoperant and discrete-trial runway extinction. Porter \& Hug (1965) suggested that the differential extinction behavior observed in the two response situations was due to the relative absence of CRs during free-operant extinction. These authors suggested that the pretraining necessary to get Ss to bar-press was sufficient to extinguish any CRs before training began-particularly when a series of bar-presses were required for reinforcement. Thus the CRs which would normally occur in free-operant extinction would be absent because these responses had been extinguished during pretraining. Consequently, groups given large numbers of reinforcements would be expected to extinguish more slowly than Ss that had received few reinforcements because of a higher level of frustration produced drive without CRs.

In the present study an attempt was made to manipulate the number of CRs that occurred during pretraining and acquisition by varying the difficulty of learning to bar-press. To accomplish this, the distance between bar and food magazine, and thus the ease of learning to press, was varied. Ss that ate and bar-pressed at the same location were expected to make few CRs during pretraining, and consequently many CRs during extinction. On the other hand, Ss trained with more conventional procedures, where the bar was some distance from the foodcup, were expected to make many CRs during pretraining and few CRs during extinction.

\section{Method}

Forty-eight male albino rats, 90-120 days old, were assigned to six groups of eight Ss each. The groups received either 18 or 96 reinforcements, delivered at the bar, 2 in. from the bar, or 4 in. from the bar.

The test chamber was 10 in. $\times 9$ in. $\times 7$ in. high, and had a wire mesh roof and floor. The chamber was illuminated by a 7-1/2 W bulb and was enclosed in a sound-resistant, ventilated chest. For the bar group (B), foodcup and bar were arranged to deliver pellets into the hollow center of the bar, which was located. 2 in. right of center on a side wall of the chamber, and 2 in. from the chamber floor. The mid groups (M) had the foodcup centered on the same wall, 2 in. from the bar, while the far groups (F) had the foodcup moved 2 in. further to the left of center, 4 in. from the bar. The $.097 \mathrm{~g}$ Noyes pellet rewards were delivered by a Davis PD 109 feeder.

All Ss were adapted to a $23 \mathrm{~h}$ feeding schedule seven days before pretraining. On Day 8, Ss received 20 pellets during the course of magazine training. On Day 9, Ss were shaped by first giving them 20 pellets on FR1 followed by four pellets each at FR2 and FR4, and then five pellets each at FR6 and FR7. On Day 10, acquisition began on FR8 reinforcement. The 18 reinforcement Ss recelved 12 pellets on Day 10 and six on Day 11, followed immediately by extinction to a criterion of $5 \mathrm{~min}$ without completing a FR8 chain. The 96 reinforcement groups received 45 pellets on both Days 10 and 11, and six pellets on Day 12, followed immediately by extinction to the same $5 \mathrm{~min}$ criterion.

\section{Results}

Response speeds over the final six acquisition trials were analyzed using the data from the last six of eight Ss in each group. Apparatus difficulties early in the experiment prevented analyzing the terminal acquisition data from all eight Ss in each group. Response speeds were reliably faster with 96 reinforcements compared to $18(\mathrm{~F}=14.76, \mathrm{df}=1 / 30, \mathrm{p}<.01)$, while neither locus of reinforcement nor the interaction between locus and number of reinforcements were significant.

Turning to extinction performance, Fig. 1 gives the mean number of trials to an extinction criterion 


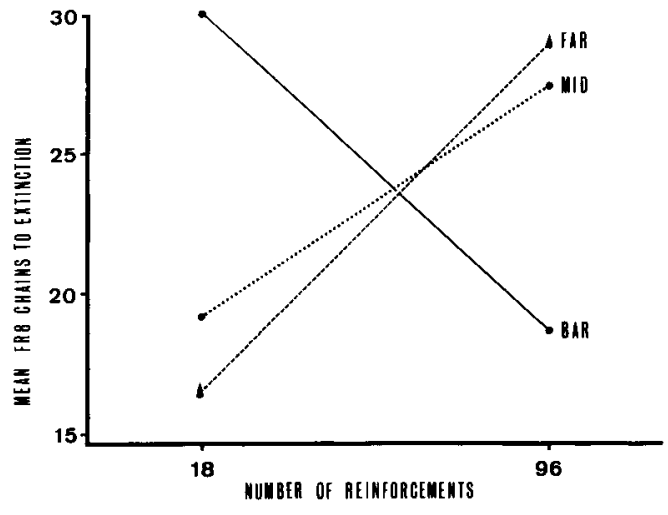

Fig. 1. Mean number of FR 8 chains to an extinction criterion of 5 min without completing a chain.

of $5 \mathrm{~min}$ without completing a FR8 chain. As the trends of Fig. 1 indicate, the interaction between locus of reinforcement and number of reinforcements was highly significant $(F=8.32, \mathrm{df}=2 / 42, \mathrm{p}<.001)$, while the main effects of these variables were not significant. Examination of the simple effects of number of reinforcements showed a significant decrease in resistance to extinction from 18 to 96 reinforcements for Group B ( $F=7.92, \mathrm{df}=1 / 14, \mathrm{p}<.025)$, while Group $F$ showed a significant increase in resistance to extinction from 18 to 96 reinforcements ( $F=5.90$, df $=1 / 14, p<.05)$. Group $M$ showed no significant change in resistance to extinction as a function of number of reinforcements, although the trend was similar to that of Group F.

The time required to reach the $5 \mathrm{~min}$ extinction criterion yielded a significant interaction between locus and number of reinforcements $(F=3.24, \mathrm{df}=$ $2 / 42, p<.05$ ), but the main effects of these variables were not reliable. Generally, the time to extinction data were less clear cut than the trials to extinction data. Simple effects tests showed only a significant change in time to extinction for the $\mathbf{B}$ group which decreased in resistance to extinction as the number of prior reinforcements increased from 18 to $96(\mathrm{~F}=29.9, \mathrm{df}=1 / 14, \mathrm{p}<.001)$.

\section{Discussion}

These results show that feeding Ss where they barpressed reversed the typical free-operant extinction pattern and yielded results similar to those obtained in the discrete-trial runway. However, Ss trained in a situation where the bar was some distance from the magazine, Groups $\mathrm{F}$ and $\mathrm{M}$, displayed a conventional free-operant increase in resistance to extinction with an increase in number of prior reinforcements.

Frustration theory can explain these results if it is assumed that: (1) Reward expectancy, and thus frustration during extinction, is stronger for all groups after 96 reinforcements than after 18 reinforcements. (2) Typical free-operant pretraining and acquisition, where bar and foodcup are separated, tends to extinguish or adapt any CRs $S$ makes. (3) The close physical proximity of bar and foodcup for Group B, and the consequent ease of learning to press, drastically reduces the number of CRs made during pretraining and acquisition. (4) These CRs are then available to Group B during extinction. (5) Groups $F$ and $M$ do not, at least over the first few trial blocks, make CRs because these responses were extinguished earlier. The frustration due to the nonreward of a previously rewarded response, then, should augment the responding of Groups $F$ and $M$ through greater drive. On the other hand, frustration should produce strong CRs in Group B and rapid extinction despite any increase in drive.

The present study demonstrated that physical proximity of manipulandum and reinforcer play a role in the extinction of free-operant behavior. However, this result should be regarded as tentative until replicated with different numbers of reinforcements and different reinforcement magnitudes. Such research is presently in progress in the author's laboratory.

\section{References}

BIRCH, D. A motivational interpretation of extinction, In $M . R$, Jones (Ed.), Nebraska Symposium on motivation. Lincoln: Nebraska University Press, 1961.

ISON, J. R. Experimental extinction as a function of number of reinforcements. J. exp. Psychol., 1962, 64, 314317.

ISON, J. R., \& COOK, P. E. Extinction performance as a function of incentive magnitude and number of acquisition trials. Psychon. Sci, 1964, 1, 245-246.

NORTH, A. J., \& STIMMEL, D. T. Extinction of an instrumental response following a large number of reinforcements. Psychol. Rep., 1960, 6, 227-234.

PERIN, C. T. Behavior potentiality as a joint function of the amount of training and the degree of hunger at the time of extinction. $J$. exp. Psychol., 1942, 30, 93-113.

PORTER, J. J., \& HUG, J. J. Discrete-trial bar-pressing performance as a function of length of response chain, number of reinforcements, and percentage reward. Psychol. Rep., 1965, 16, 1109-1116.

WILLIAMS, S. B. Resistance to extinction as a function of the number of reinforcements. J. exp. Psychol, 1938, 23, 506-522.

\section{Note}

1. This research was supported by NSF Grant GB-3466 to the author. 